J. Nonlinear Var. Anal. 5 (2021), No. 6, pp. 909-927

Available online at http://jnva.biemdas.com

https://doi.org/10.23952/jnva.5.2021.6.05

\title{
A SET SCALARIZATION FUNCTION AND DINI DIRECTIONAL DERIVATIVES WITH APPLICATIONS IN SET OPTIMIZATION PROBLEMS
}

\author{
YU HAN ${ }^{1}$, NAN-JING HUANG ${ }^{2, *}$, CHING-FENG WEN ${ }^{3,4}$ \\ ${ }^{1}$ School of Statistics, Jiangxi University of Finance and Economics, Nanchang, Jiangxi 330013, China \\ ${ }^{2}$ Department of Mathematics, Sichuan University, Chengdu, Sichuan 610064, China \\ ${ }^{3}$ Center for Fundamental Science, and Research Center for Nonlinear Analysis and Optimization, \\ Kaohsiung Medical University, Kaohsiung 80708, Taiwan \\ ${ }^{4}$ Department of Medical Research, Kaohsiung Medical University Hospital, Kaohsiung 80708, Taiwan
}

\begin{abstract}
In this paper, based on the oriented distance function of Hiriart-Urruty, we investigate a set scalarization function of sup-inf type. Some properties of the set scalarization function allow us to define the Dini directional derivatives for set-valued mappings, which is an extension of the classical Dini derivatives for scalar functions. We also obtain some properties of the Dini directional derivatives for set-valued mappings. As applications, we derive some necessary and sufficient optimality conditions for set optimization problems.
\end{abstract}

Keywords. Dini directional derivative; Oriented distance function; Optimality condition; Set optimization problem; Set scalarization function.

\section{INTRODUCTION}

Set optimization problems are a very rich class of decision-making problems. Because setvalued mappings appear naturally in many practical problems, set optimization problems will remain an important and active research topic in both the near and foreseeable future [1]. As pointed out by Kim et al. [2], studying set optimization problems is at the core of most recent developments in multiobjective optimization and practical applications. In recent years, set optimization problems have received an increasing attention due to extensive applications in many areas, such as optimal control problems, vector variational inequalities, vector optimization problems, fuzzy optimization problems, viability theory, image processing problems, mathematical economics and differential inclusions. For a detailed introduction to set optimization and its applications, we refer to [1], and for the applications in finance, we refer to [3]. For some related topics, we refer to $[4,5,6,7]$ and the references therein.

The theory and the methods of scalarization have always been of the utmost importance in vecter optimization problems $[8,9,10,11]$ and in set optimization problems $[12,13,14,15,16$,

\footnotetext{
${ }^{*}$ Corresponding author.

E-mail addresses: hanyumath@163.com (Y. Han), nanjinghuang@hotmail.com (N.J. Huang), cfwen@kmu. edu.tw (C.-F. Wen)

Received June 3, 2021; Accepted August 15, 2021.
}

(C)2021 Journal of Nonlinear and Variational Analysis 
17] from theoretical as well as computational points of view. The linear scalarization is historically the first method proposed and the most widely known and used. There are two important types of nonlinear scalarization functions. The first one is Gerstewitz's function [18], which has some nice properties, especially monotonicity properties and nonconvex separation properties $[8,10,19,20,21]$. It provides an important tool in the study of many fundamental mathematical problems, such as, the vector optimization and the vector equilibrium problems. The second one is the oriented distance function of Hiriart-Urruty, which was introduced in [22, 23] to analyze the geometry of nonsmooth optimization problems and obtain necessary optimality conditions. It has been applied to establish the nonlinear scalarization results, Lagrange multiplier rules and the well-posedness results for vector optimization problems [24, 25, 26, 27, 28]. The oriented distance function of Hiriart-Urruty has also been employed to the study of set optimization problems. For example, Chen, Ansari, and Yao [29] obtained some characterizations of various set order relations by using the oriented distance function, and then derived necessary and sufficient conditions for four types of optimal solutions of constrained set optimization problem with respect to the set order relations. Ansari, Köbis, and Sharma [30] gave some characterizations of set relations with respect to variable domination structures by defining scalarization functions in terms of the oriented distance function and derived the characterization of minimal elements of a family of sets and the properties of sets of minimal elements. Ha [31] introduced a set scalarization function of sup-inf type, which is an extension of the oriented distance function of Hiriart-Urruty. By employing the set scalarization function, the author of [31] defined a generalized Hausdorff-type distance and a directional derivative for set-valued mappings, and derived necessary and/or sufficient conditions for various minimizers and maximizers of setvalued mappings. Jiménez, Novo, and Vílchez [32, 33] obtained several interesting properties of the set scalarization function of sup-inf type introduced by Ha [31] and applied them to characterize several concepts of minimal solution to a set optimization problem. Jiménez, Novo, and Vílchez [34] investigated six set scalarization functions, which are extensions of the oriented distance of Hiriart-Urruty. They also derived several necessary and sufficient minimality conditions for set optimization problems by using the six set scalarization functions.

It is worth noting that the set scalarization function introduced by Ha [31] may be negative. However, the authors in [31, 32, 33, 34] only discussed some properties of the set scalarization function with nonnegative values. What happens to the set scalarization function if it is negative? The first aim of this paper is to derive some properties of the set scalarization function with negative values. On the other hand, it is well known that the derivative and directional derivatives play a critical role for establishing optimality conditions in optimization theory. Very recently, Han [35] introduced the Clarke generalized directional derivative for set-valued mappings by using the nonlinear scalarizing function introduced by Hernández and RodríguezMarín [14], and obtained some properties of the Clarke generalized directional derivative. As applications, the author of [35] presented necessary and sufficient optimality conditions for set optimization problems. We note that some properties of the set scalarization function allow us to define the Dini directional derivatives for set-valued mappings, which is an extension of the classical Dini derivatives for scalar functions [36]. Thus, one natural question is: can we employ the Dini directional derivatives for set-valued mappings to capture optimality conditions for set optimization problems? The second aim of this paper is to obtain some properties of the 
Dini directional derivatives for set-valued mappings and apply them to derive some necessary and sufficient optimality conditions for set optimization problems.

The rest of the paper is organized as follows. The next section presents some necessary notations and lemmas. In Section 3, we investigate a set scalarization function via oriented distance function introduced by $\mathrm{Ha}$ [31]. Before summarizing this paper in Section 5, we study the Dini directional derivatives for set-valued mappings and employ them to obtain some necessary and sufficient optimality conditions for set optimization problems in Section 4.

\section{PRELIMINARIES}

Throughout this paper, let $X$ and $Y$ be two normed vector spaces. We denote by $B_{Y}$ and $B_{Y}^{0}$ the closed unit ball and the open unit ball in $Y$, respectively. Let $\wp_{0}(Y)$ be the family of all nonempty subsets of Y. Assume that $K \subseteq Y$ is a convex, closed and pointed cone with nonempty interior. The cone $K$ induces a partial order on $Y$ as follows: for $a, b \in Y$,

$$
a \leq b: \Leftrightarrow b-a \in K .
$$

Let $Y^{*}$ be the topological dual space of $Y$, and let $K^{*}$ be the topological dual cone of $K$, defined by

$$
K^{*}=\left\{f \in Y^{*}: f(y) \geq 0, \forall y \in K\right\} .
$$

Let $B_{K}^{*}=\left\{f \in K^{*}:\|f\|=1\right\}$, and, for $A \in \wp_{0}(Y)$ and $f \in Y^{*}$, let $S(f, A)=\sup _{a \in A} f(a)$ be the support function of $A$ at $f$. Let $A, B \in \wp_{0}(Y)$. The lower relation " $\leq l$ " and the weak lower relation "«l", respectively, are defined by

$$
A \leq^{l} B \Leftrightarrow B \subseteq A+K \text { and } A \ll^{l} B \Leftrightarrow B \subseteq A+\operatorname{int} K .
$$

Denote $A \sim^{l} B$ iff $A \leq{ }^{l} B$ and $B \leq{ }^{l} A$. We denote by int $A, \operatorname{cl} A, \partial A, \operatorname{co} A$, and $A^{c}$ the topological interior, the topological closure, the topological boundary, the convex hull, and the complementary set of $A$, respectively. It is said that a nonempty set $A \subseteq Y$ is $K$-proper if $A+K \neq Y$, $K$-convex if $A+K$ is a convex set, $K$-closed if $A+K$ is a closed set, $K$-bounded if for each neighborhood $U$ of zero in $Y$ there is some positive number $t$ such that $A \subseteq t U+K$, and $K$ compact if any cover of $A$ of the form $\left\{U_{\alpha}+K: U_{\alpha}\right.$ are open $\}$ admits a finite subcover. The family of the neighborhoods of $0 \in Y$ is denoted by $N(0)$.

Remark 2.1. Clearly, if there exists $\beta>0$ such that $\beta B_{Y}$ is $K$-closed, then $\delta B_{Y}$ is $K$-closed for any $\delta>0$.

Let $A \in \wp_{0}(Y)$ and $a \in A$. We say that $a$ is a minimal point of $A$ with respect to $K$, denoted by $a \in \operatorname{Min}(A)$, if $(A-a) \cap(-K)=\{0\}$. We say that $a$ is a weak minimal point of $A$ with respect to $K$ and we write $a \in \mathrm{WMin}(A)$, if $(A-a) \cap(-\operatorname{int} K)=\emptyset$.

Remark 2.2. Obviously, $\operatorname{Min}(A) \subseteq \mathrm{WMin}(A)$. Moreover, if $A$ is nonempty and $K$-compact, then $\operatorname{Min}(A) \neq \emptyset$ (see [10]).

Let $F: X \rightrightarrows Y$ be a set-valued mapping, and let $D$ be a nonempty subset of $X$. We consider the following set optimization problem:

$$
\text { (SOP) } \min F(x) \quad \text { subject to } \quad x \in D \text {. }
$$

Definition 2.1. An element $x_{0} \in D$ is said to be

(i) $l$-minimal solution of (SOP) if, for $x \in D, F(x) \leq^{l} F\left(x_{0}\right)$ implies that $F\left(x_{0}\right) \leq{ }^{l} F(x)$; 
(ii) weak $l$-minimal solution of (SOP) if, for $x \in D, F(x) \ll^{l} F\left(x_{0}\right)$ implies that $F\left(x_{0}\right) \ll^{l} F(x)$.

Let $E_{l}(F, D)$ and $W_{l}(F, D)$ denote the $l$-minimal solution set and the weak $l$-minimal solution set of (SOP), respectively.

Definition 2.2. [37] Let $D$ be a nonempty convex subset of $X$. A set-valued mapping $\Phi: X \rightrightarrows Y$ is said to be $K$-convex on $D$ if, for any $x_{1}, x_{2} \in D$ and for any $t \in[0,1]$,

$$
t \Phi\left(x_{1}\right)+(1-t) \Phi\left(x_{2}\right) \subseteq \Phi\left(t x_{1}+(1-t) x_{2}\right)+K .
$$

Definition 2.3. [38] Let $(X, d)$ be a metric space, and let $A$ and $B$ be two nonempty subsets of $X$. The Hausdorff distance between $A$ and $B$ is defined by

$$
H(A, B):=\max \{e(A, B), e(B, A)\},
$$

where

$$
e(A, B):=\sup _{a \in A} d(a, B), \quad d(a, B):=\inf _{b \in B} d(a, b) .
$$

Definition 2.4. [22] For a set $A \subseteq Y$, let the oriented distance function $\Delta_{A}: Y \rightarrow \mathbb{R} \cup\{ \pm \infty\}$ be defined as

$$
\Delta_{A}(y)=d_{A}(y)-d_{Y \backslash A}(y)
$$

with $d_{\emptyset}(y)=+\infty$, where $d_{A}(y)=\inf _{x \in A}\|y-x\|$.

Next, we collect the basic properties of the oriented distance function.

Lemma 2.1. $[1,17,28]$ If $A \subseteq Y$ is nonempty and $A \neq Y$, then

(i) $\Delta_{A}$ is real valued;

(ii) $\Delta_{A}$ is 1-Lipschitzian;

(iii) $\Delta_{A}(y)<0 \Leftrightarrow y \in \operatorname{int} A$;

(iv) $\Delta_{A}(y)=0 \Leftrightarrow y \in \partial A$;

(v) $\Delta_{A}(y)>0 \Leftrightarrow y \in \operatorname{int} A^{c}$;

(vi) if $A$ is closed, then it holds that $A=\left\{y \in Y: \Delta_{A}(y) \leq 0\right\}$;

(vii) $\Delta_{A}$ is positively homogeneous providing $A$ is a cone;

(viii) $\Delta_{A}$ is convex providing $A$ is convex;

(ix) if $A$ is a closed and convex cone, then $\Delta_{A}$ is nonincreasing with respect to the ordering relation induced on $Y$, i.e., for any $y_{1}, y_{2} \in Y$,

$$
y_{1}-y_{2} \in A \Rightarrow \Delta_{A}\left(y_{1}\right) \leq \Delta_{A}\left(y_{2}\right) ;
$$

if $A$ has a nonempty interior, then for any $y_{1}, y_{2} \in Y$,

$$
y_{1}-y_{2} \in \operatorname{int} A \Rightarrow \Delta_{A}\left(y_{1}\right)<\Delta_{A}\left(y_{2}\right) .
$$

It is easy to have the following lemma.

Lemma 2.2. Let $\delta>0$. Then $d_{A}(y) \geq \delta \Leftrightarrow\left(y+\delta B_{Y}^{0}\right) \cap A=\emptyset$.

Lemma 2.3. Let $\delta \geq 0$. Then the following statements are true:

(i) if $y \in \delta B_{Y}+A$, then $d_{A}(y) \leq \delta$;

(ii) if $\delta B_{Y}+A$ is closed and $d_{A}(y) \leq \delta$, then $y \in \delta B_{Y}+A$. 
Proof. (i). The conclusion is trivial.

(ii). For any $n \in \mathbb{N}$, it follows from $d_{A}(y) \leq \delta$ that $d_{A}(y) \leq \delta<\delta+\frac{1}{n}$. Then there exists $a_{n} \in A$ such that $\left\|y-a_{n}\right\|<\delta+\frac{1}{n}$. Consequently,

$$
y \in\left(\delta+\frac{1}{n}\right) B_{Y}+a_{n} \subseteq\left(\delta+\frac{1}{n}\right) B_{Y}+A, \quad \forall n \in \mathbb{N} .
$$

Thus, there exists $b_{n} \in B_{Y}$ such that $y+\frac{1}{n} b_{n} \in \delta B_{Y}+A$. Since $y+\frac{1}{n} b_{n} \rightarrow y$ and $\delta B_{Y}+A$ is closed, we have $y \in \delta B_{Y}+A$.

From Remark 2.1 and Lemma 2.3, we can obtain the following corollary.

Corollary 2.1. If $A$ is a cone and $B_{Y}+A$ is closed, then, for any $\delta>0, d_{A}(y) \leq \delta \Rightarrow y \in$ $\delta B_{Y}+A$.

Lemma 2.4. If $\delta>0$ and $A$ is $K$-bounded, then $A \not \subset A+\bigcap_{\beta \in \delta B_{Y}}(\beta+\operatorname{int} K)$.

Proof. Suppose that

$$
A \subseteq A+\bigcap_{\beta \in \delta B_{Y}}(\beta+\operatorname{int} K) .
$$

Then, for any given $x_{1} \in A$, it follows from (2.1) that there exists $x_{2} \in A$ such that

$$
x_{1}-x_{2} \in \bigcap_{\beta \in \delta B_{Y}}(\beta+\operatorname{int} K) .
$$

For any $\beta \in \delta B_{Y}$, it is clear that $-\beta \in \delta B_{Y}$. This together with (2.2) implies that $x_{1}-x_{2} \in-\beta+$ int $K$ and so $x_{1}-x_{2}+\beta \in \operatorname{int} K$. By the arbitrariness of $\beta \in \delta B_{Y}$, we have $x_{1}-x_{2}+\delta B_{Y} \subseteq \operatorname{int} K$. By the similar arguments, for $x_{n} \in A$, there exists $x_{n+1} \in A$ such that $x_{n}-x_{n+1}+\delta B_{Y} \subseteq \operatorname{int} K$. This shows that $x_{1}-x_{n+1}+n \delta B_{Y} \subseteq \operatorname{int} K$ and so

$$
x_{1}-x_{n+1}+n \delta B_{Y}+K \subseteq \operatorname{int} K+K \subseteq \operatorname{int} K .
$$

Since $A$ is $K$-bounded, there exists $\xi>0$ such that $A \subseteq \xi B_{Y}+K$. It is clear that there exists $n_{0}$ large enough such that $n_{0} \delta>\left\|x_{1}\right\|+\xi$. Noting that $x_{n_{0}+1} \in A \subseteq \xi B_{Y}+K$, there exist $b_{0} \in \xi B_{Y}$ and $k_{0} \in K$ such that $x_{n_{0}+1}=b_{0}+k_{0}$. Due to $-x_{1}+b_{0} \in n_{0} \delta B_{Y}, k_{0} \in K$ and (2.3), we have

$$
0=x_{1}-x_{n_{0}+1}+\left(-x_{1}+b_{0}\right)+k_{0} \in x_{1}-x_{n_{0}+1}+n_{0} \delta B_{Y}+K \subseteq \operatorname{int} K,
$$

which is a contradiction.

From Lemma 2.6 of [14] and Remark 2.2, we can obtain the following lemma.

Lemma 2.5. If $x_{0} \in D$ and $F\left(x_{0}\right)$ is nonempty and $K$-compact, then $x_{0} \in W_{l}(F, D)$ if and only if there does not exist $y \in D$ satisfying $F(y) \ll^{l} F\left(x_{0}\right)$.

\section{A Set Scalarization Function via Oriented Distance Function}

In this section, we study the set scalarization function of sup-inf type introduced by $\mathrm{Ha}$ [31], which is an extension of the oriented distance function of Hiriart-Urruty.

Let $A$ and $B$ be nonempty subsets of $Y$. Ha [31] introduced the following scalarization function:

$$
h_{K}(A, B)=\sup _{b \in B} \inf _{a \in A} \Delta_{-K}(a-b) .
$$

Lemma 3.1. [31] Let $A$ and $B$ be nonempty subsets of $Y$. 
(i) If $A$ is $K$-bounded, then $h_{K}(A, B)>-\infty$.

(ii) If $B$ is $K$-bounded, then $h_{K}(A, B)<+\infty$.

Lemma 3.2. [31] Let $A$ and $B$ be nonempty subsets of $Y$ and $y \in Y$.

(i) If $A$ is $K$-compact, then there exists $a_{0} \in A$ such that

$$
\Delta_{-K}\left(a_{0}-y\right)=\inf _{a \in A} \Delta_{-K}(a-y) .
$$

(ii) If $A$ is $K$-bounded and $B$ is $K$-compact, then there exists $b_{0} \in B$ such that

$$
h_{K}(A, B)=\inf _{a \in A} \Delta_{-K}\left(a-b_{0}\right) .
$$

From Theorem 5.1 of [32], we can obtain the following proposition.

Proposition 3.1. Let $\delta \geq 0$. The following statements are true:

(i) if $B \subseteq A+K+\delta B_{Y}$, then $h_{K}(A, B) \leq \delta$;

(ii) if $A+K+\delta B_{Y}$ is closed and $h_{K}(A, B) \leq \delta$, then $B \subseteq A+K+\delta B_{Y}$.

From Proposition 3.1, we can obtain the following corollary.

Corollary 3.1. [33] The following statements are true:

(i) if $A \leq{ }^{l} B$, then $h_{K}(A, B) \leq 0$;

(ii) if $A$ is $K$-closed and $h_{K}(A, B) \leq 0$, then $A \leq l B$.

Remark 3.1. Corollary 3.1 improves Lemma 3.3 of [31].

Proposition 3.2. Let $\delta \geq 0$. Then the following statements are true:

(i) if $h_{K}(A, B)<\delta$, then $B \subseteq A+K+\delta B_{Y}^{0}$;

(ii) if $A$ is $K$-bounded, $B$ is $K$-compact and $B \subseteq A+K+\delta B_{Y}^{0}$, then $h_{K}(A, B)<\delta$.

Proof. (i). For any $b \in B$, it follows from $h_{K}(A, B)<\delta$ that $\inf _{a \in A} \Delta_{-K}(a-b)<\delta$. Then there exists $a_{0} \in A$ such that $\Delta_{-K}\left(a_{0}-b\right)<\delta$. There are two cases to be considered.

Case 1. $a_{0}-b \in-K$. Then $b \in a_{0}+K \subseteq A+K \subseteq A+K+\delta B_{Y}^{0}$.

Case 2. $a_{0}-b \notin-K$. Thus, $\Delta_{-K}\left(a_{0}-b\right)=d_{-K}\left(a_{0}-b\right)<\delta$. Due to Lemma 2.2, we have $a_{0}-b \in-K+\delta B_{Y}^{0}$, and so $b \in a_{0}+K+\delta B_{Y}^{0} \subseteq A+K+\delta B_{Y}^{0}$.

By the arbitrariness of $b \in B$, we have $B \subseteq A+K+\delta B_{Y}^{0}$.

(ii). In view of Lemma 3.2 (ii), there exists $b_{0} \in B$ such that

$$
h_{K}(A, B)=\inf _{a \in A} \Delta_{-K}\left(a-b_{0}\right) .
$$

Due to $B \subseteq A+K+\delta B_{Y}^{0}$, there exists $a_{0} \in A$ such that $b_{0}-a_{0} \in K+\delta B_{Y}^{0}$, and so $a_{0}-b_{0} \in$ $-K+\delta B_{Y}^{0}$. By Lemma 2.2, we have $\Delta_{-K}\left(a_{0}-b_{0}\right) \leq d_{-K}\left(a_{0}-b_{0}\right)<\delta$. It follows from (3.1) that

$$
h_{K}(A, B)=\inf _{a \in A} \Delta_{-K}\left(a-b_{0}\right) \leq \Delta_{-K}\left(a_{0}-b_{0}\right)<\delta .
$$

This completes the proof.

Proposition 3.3. Let $\delta>0$. Then the following statements are true:

(i) if $B \subseteq A+\bigcap_{\beta \in \delta B_{Y}^{0}}(\beta+K)$, then $h_{K}(A, B) \leq-\delta$;

(ii) if $A$ is $K$-compact and $h_{K}(A, B) \leq-\delta$, then $B \subseteq A+\bigcap_{\beta \in \delta B_{Y}^{0}}(\beta+K)$. 
Proof. (i). For any $b \in B$, due to $B \subseteq A+\bigcap_{\beta \in \delta B_{Y}^{0}}(\beta+K)$, there exists $\bar{a} \in A$ such that $b-$ $\bar{a} \in \bigcap_{\beta \in \delta B_{Y}^{0}}(\beta+K)$. This means that for any $\beta \in \delta B_{Y}^{0}$, we have $b-\bar{a} \in \beta+K$, and so $\bar{a}-$ $b+\beta \in-K$. By the arbitrariness of $\beta \in \delta B_{Y}^{0}$, we have $\bar{a}-b+\delta B_{Y}^{0} \in-K$. Consequently, $\left(\bar{a}-b+\delta B_{Y}^{0}\right) \cap(Y \backslash(-K))=\emptyset$. It follows from Lemma 2.2 that $d_{Y \backslash(-K)}(\bar{a}-b) \geq \delta$. It is clear that $\bar{a}-b \in-K$. Thus,

$$
\Delta_{-K}(\bar{a}-b)=-d_{Y \backslash(-K)}(\bar{a}-b) \leq-\delta,
$$

and so

$$
\inf _{a \in A} \Delta_{-K}(a-b) \leq \Delta_{-K}(\bar{a}-b) \leq-\delta, \quad \forall b \in B .
$$

By the arbitrariness of $b \in B$, we have

$$
h_{K}(A, B)=\sup _{b \in B} \inf _{a \in A} \Delta_{-K}(a-b) \leq-\delta .
$$

(ii). For any given $b \in B$, it follows from $h_{K}(A, B) \leq-\delta$ that $\inf _{a \in A} \Delta_{-K}(a-b) \leq-\delta$. Since $A$ is $K$-compact, by Lemma 3.2 (i), there exists $a_{0} \in A$ such that

$$
\Delta_{-K}\left(a_{0}-b\right)=\inf _{a \in A} \Delta_{-K}(a-b) \leq-\delta<0 .
$$

Then $a_{0}-b \in-K$ and $\Delta_{-K}\left(a_{0}-b\right)=-d_{Y \backslash(-K)}\left(a_{0}-b\right) \leq-\delta$, and so

$$
d_{Y \backslash(-K)}\left(a_{0}-b\right) \geq \delta .
$$

Due to Lemma 2.2, we have $\left(a_{0}-b+\delta B_{Y}^{0}\right) \cap(Y \backslash(-K))=\emptyset$, which means that $a_{0}-b+\delta B_{Y}^{0} \in$ $-K$. Consequently, for any $\beta \in \delta B_{Y}^{0}$, we have $a_{0}-b+\beta \in-K$, and so $b-a_{0} \in \beta+K$. Due to the arbitrariness of $\beta \in \delta B_{Y}^{0}$, we have $b-a_{0} \in \bigcap_{\beta \in \delta B_{Y}^{0}}(\beta+K)$. Hence,

$$
b \in a_{0}+\bigcap_{\beta \in \delta B_{Y}^{0}}(\beta+K) \subseteq A+\bigcap_{\beta \in \delta B_{Y}^{0}}(\beta+K) .
$$

It follows from the arbitrariness of $b \in B$ that $B \subseteq A+\bigcap_{\beta \in \delta B_{Y}^{0}}(\beta+K)$.

Proposition 3.4. Let $\delta>0$. Then the following statements are true:

(i) if $h_{K}(A, B)<-\delta$, then $B \subseteq A+\bigcap_{\beta \in \delta B_{Y}}(\beta+\operatorname{int} K)$;

(ii) if $A$ is $K$-bounded, $B$ is $K$-compact, $Y$ is finite dimensional and $B \subseteq A+\bigcap_{\beta \in \delta B_{Y}}(\beta+\operatorname{int} K)$, then $h_{K}(A, B)<-\delta$.

Proof. (i). For any $b \in B$, it follows from $h_{K}(A, B)<-\delta$ that $\inf _{a \in A} \Delta_{-K}(a-b)<-\delta$. Then there exists $a_{0} \in A$ such that $\Delta_{-K}\left(a_{0}-b\right)<-\delta<0$. This means that

$$
\Delta_{-K}\left(a_{0}-b\right)=-d_{Y \backslash(-K)}\left(a_{0}-b\right)<-\delta .
$$

Thus, $d_{Y \backslash(-K)}\left(a_{0}-b\right)>\delta$. Thus, there exists $\eta \in \mathbb{R}$ such that

$$
d_{Y \backslash(-K)}\left(a_{0}-b\right)>\eta>\delta .
$$

By Lemma 2.3 (i), we have

$$
\left(a_{0}-b+\eta B_{Y}\right) \cap(Y \backslash(-K))=\emptyset,
$$

which implies that

$$
a_{0}-b+\eta B_{Y}=a_{0}-b+\delta B_{Y}+(\eta-\delta) B_{Y} \subseteq-K .
$$


It follows that $a_{0}-b+\delta B_{Y} \subseteq-\operatorname{int} K$. For any $\beta \in \delta B_{Y}$, we have $a_{0}-b+\beta \in-\operatorname{int} K$, and so $b-a_{0} \in \beta+\operatorname{int} K$. Due to the arbitrariness of $\beta \in \delta B_{Y}$, we have $b-a_{0} \in \bigcap_{\beta \in \delta B_{Y}}(\beta+\operatorname{int} K)$. Consequently,

$$
b \in a_{0}+\bigcap_{\beta \in \delta B_{Y}}(\beta+\operatorname{int} K) \subseteq A+\bigcap_{\beta \in \delta B_{Y}}(\beta+\operatorname{int} K),
$$

By the arbitrariness of $b \in B$, we have $B \subseteq A+\bigcap_{\beta \in \delta B_{Y}}(\beta+\operatorname{int} K)$.

(ii). It follows from Lemma 3.2 (ii) that there exists $b_{0} \in B$ such that

$$
h_{K}(A, B)=\inf _{a \in A} \Delta_{-K}\left(a-b_{0}\right) .
$$

Due to $B \subseteq A+\bigcap_{\beta \in \delta B_{Y}}(\beta+\operatorname{int} K)$, there exists $a_{0} \in A$ such that

$$
b_{0}-a_{0} \in \bigcap_{\beta \in \delta B_{Y}}(\beta+\operatorname{int} K) .
$$

This means that $b_{0}-a_{0}+\delta B_{Y} \subseteq \operatorname{int} K$, and so $a_{0}-b_{0}+\delta B_{Y} \subseteq-\mathrm{int} K$. Since $Y$ is finite dimensional, we obtain that $a_{0}-b_{0}+\delta B_{Y}$ is compact. Then there exists $\xi>0$ such that $a_{0}-b_{0}+(\delta+\xi) B_{Y} \subseteq-K$, which implies that

$$
\left(a_{0}-b_{0}+(\delta+\xi) B_{Y}^{0}\right) \cap(Y \backslash(-K))=\emptyset .
$$

In view of Lemma 2.2, we have $d_{Y \backslash(-K)}\left(a_{0}-b_{0}\right) \geq \delta+\xi>\delta$. Consequently,

$$
\Delta_{-K}\left(a_{0}-b_{0}\right)=-d_{Y \backslash(-K)}\left(a_{0}-b_{0}\right)<-\delta .
$$

This together with (3.2) implies that

$$
h_{K}(A, B)=\inf _{a \in A} \Delta_{-K}\left(a-b_{0}\right) \leq \Delta_{-K}\left(a_{0}-b_{0}\right)<-\delta .
$$

This completes the proof.

Theorem 3.1. Assume that $A$ and $B$ are $K$-bounded.

(i) If $h_{K}(A, B) \geq 0$, then $h_{K}(A, B)=\inf \left\{t \geq 0: B \subseteq A+K+t B_{Y}\right\}$.

(ii) If $h_{K}(A, B)<0$, then $h_{K}(A, B)=\inf \left\{t<0: B \subseteq A+\bigcap_{\beta \in(-t) B_{Y}}(\beta+K)\right\}$.

Proof. (i). Since $B$ is $K$-bounded, in view of Lemma 3.1 (ii), we have $h_{K}(A, B)<+\infty$. Then there exists $\varphi>0$ such that $h_{K}(A, B)<\varphi$. It follows from Proposition 3.2 (i) that

$$
B \subseteq A+K+\varphi B_{Y}^{0} \subseteq A+K+\varphi B_{Y},
$$

which means that $\left\{t \geq 0: B \subseteq A+K+t B_{Y}\right\} \neq \emptyset$. Let $\eta=\inf \left\{t \geq 0: B \subseteq A+K+t B_{Y}\right\}$. For any $\varepsilon>0$, there exists $t \geq 0$ such that $B \subseteq A+K+t B_{Y}$ and $t<\eta+\varepsilon$. Due to Proposition 3.1 (i), we have $h_{K}(A, B) \leq t<\eta+\varepsilon$. By the arbitrariness of $\varepsilon>0$, we have $h_{K}(A, B) \leq \eta$.

On the other hand, suppose that $h_{K}(A, B)<\eta$. Then there exists $\beta \in \mathbb{R}$ such that

$$
h_{K}(A, B)<\beta<\eta \text {. }
$$

It follows from Proposition 3.2 (i) that $B \subseteq A+K+\beta B_{Y}^{0} \subseteq A+K+\beta B_{Y}$. This implies that $\eta \leq \beta$, which contradicts (3.3). Therefore, $h_{K}(A, B) \geq \eta$.

(ii). Due to $h_{K}(A, B)<0$, there exists $\delta \in \mathbb{R}$ such that $h_{K}(A, B)<\delta<0$. Applying Proposition 3.4 (i), we have

$$
B \subseteq A+\bigcap_{\beta \in(-\delta) B_{Y}}(\beta+\operatorname{int} K) \subseteq A+\bigcap_{\beta \in(-\delta) B_{Y}}(\beta+K),
$$


which means that $\left\{t<0: B \subseteq A+\bigcap_{\beta \in(-t) B_{Y}}(\beta+K)\right\} \neq \emptyset$. Suppose that

$$
\inf \left\{t<0: B \subseteq A+\bigcap_{\beta \in(-t) B_{Y}}(\beta+K)\right\}=-\infty \text {. }
$$

Noting that $A$ is $K$-bounded, by Lemma 3.1 (i), we have $h_{K}(A, B)>-\infty$. Then there exists $\xi<0$ such that

$$
h_{K}(A, B)>\xi .
$$

It follows from (3.4) that inf $\left\{t<0: B \subseteq A+\bigcap_{\beta \in(-t) B_{Y}}(\beta+K)\right\}<\xi$. Consequently, there exists $t_{0}<0$ with $t_{0}<\xi$ such that

$$
B \subseteq A+\bigcap_{\beta \in\left(-t_{0}\right) B_{Y}}(\beta+K) \subseteq A+\bigcap_{\beta \in\left(-t_{0}\right) B_{Y}^{0}}(\beta+K) .
$$

In view of Proposition 3.3 (i), we have $h_{K}(A, B) \leq t_{0}<\xi$, which contradicts (3.5). Therefore,

$$
\eta:=\inf \left\{t<0: B \subseteq A+\bigcap_{\beta \in(-t) B_{Y}}(\beta+K)\right\}>-\infty .
$$

For any $\varepsilon>0$, there exists $t<0$ with $t<\eta+\varepsilon$ such that

$$
B \subseteq A+\bigcap_{\beta \in(-t) B_{Y}}(\beta+K) \subseteq A+\bigcap_{\beta \in(-t) B_{Y}^{0}}(\beta+K) .
$$

Applying Proposition 3.3 (i), we have $h_{K}(A, B) \leq t<\eta+\varepsilon$. Due to the arbitrariness of $\varepsilon>0$, we have $h_{K}(A, B) \leq \eta$. Suppose that $h_{K}(A, B)<\eta$. Then there exists $\phi \in \mathbb{R}$ such that

$$
h_{K}(A, B)<\phi<\eta \text {. }
$$

We conclude from (3.6) and Proposition 3.4 (i) that

$$
B \subseteq A+\bigcap_{\beta \in(-\phi) B_{Y}}(\beta+\text { int } K) \subseteq A+\bigcap_{\beta \in(-\phi) B_{Y}}(\beta+K) .
$$

This means that $\eta \leq \phi$, which contradicts (3.6). Therefore, we have $h_{K}(A, B) \geq \eta$.

Lemma 3.3. The following statements are true:

(i) If $\xi>0$ and $\delta>0$, then

$$
\bigcap_{\beta \in \xi \delta B_{Y}}(\beta+K)=\xi\left(\bigcap_{\beta \in \delta B_{Y}}(\beta+K)\right), \quad \bigcap_{\beta \in \xi \delta B_{Y}}(\beta+\operatorname{int} K)=\xi\left(\bigcap_{\beta \in \delta B_{Y}}(\beta+\operatorname{int} K)\right) \text {. }
$$

(ii) If $\delta_{1}>0$ and $\delta_{2}>0$, then

$$
\bigcap_{\beta \in \delta_{1} B_{Y}}(\beta+K)+\bigcap_{\beta \in \delta_{2} B_{Y}}(\beta+K) \subseteq \bigcap_{\beta \in\left(\delta_{1}+\delta_{2}\right) B_{Y}}(\beta+K) .
$$

(iii) If $\delta_{2} \geq \delta_{1}>0$, then

$$
\bigcap_{\beta \in \delta_{1} B_{Y}}(\beta+K)+\delta_{2} B_{Y}+K \subseteq\left(\delta_{2}-\delta_{1}\right) B_{Y}+K
$$


(iv) If $0 \leq \delta_{2}<\delta_{1}$, then

$$
\bigcap_{\beta \in \delta_{1} B_{Y}}(\beta+K)+\delta_{2} B_{Y}+K \subseteq \bigcap_{\beta \in\left(\delta_{1}-\delta_{2}\right) B_{Y}}(\beta+K) .
$$

Proof. (i). The conclusion is trivial.

(ii). Let $z_{i} \in \bigcap_{\beta \in \delta_{i} B_{Y}}(\beta+K), i=1,2$. Then for any $\beta_{i} \in \delta_{i} B_{Y}$, we have

$$
z_{i} \in \beta_{i}+K, \quad i=1,2 .
$$

For any $u \in\left(\delta_{1}+\delta_{2}\right) B_{Y}$, it is clear that $\frac{\delta_{1}}{\delta_{1}+\delta_{2}} u \in \delta_{1} B_{Y}$ and $\frac{\delta_{2}}{\delta_{1}+\delta_{2}} u \in \delta_{2} B_{Y}$. It follows from (3.7) that $z_{1} \in \frac{\delta_{1}}{\delta_{1}+\delta_{2}} u+K$ and $z_{2} \in \frac{\delta_{2}}{\delta_{1}+\delta_{2}} u+K$, and so $z_{1}+z_{2} \in u+K$. By the arbitrariness of $u \in\left(\delta_{1}+\delta_{2}\right) B_{Y}$, we have $z_{1}+z_{2} \in \bigcap_{\beta \in\left(\delta_{1}+\delta_{2}\right) B_{Y}}(\beta+K)$.

(iii). Let $z \in \bigcap_{\beta \in \delta_{1} B_{Y}}(\beta+K), u \in \delta_{2} B_{Y}$ and $k_{0} \in K$. Then

$$
z \in \beta+K, \quad \forall \beta \in \delta_{1} B_{Y} .
$$

Due to $\frac{-\delta_{1}}{\delta_{2}} u \in \delta_{1} B_{Y}$ and (3.8), we have $z \in \frac{-\delta_{1}}{\delta_{2}} u+K$. Consequently,

$$
z+u+k_{0} \in \frac{-\delta_{1}}{\delta_{2}} u+K+u+k_{0} \subseteq \frac{\delta_{2}-\delta_{1}}{\delta_{2}} u+K \subseteq\left(\delta_{2}-\delta_{1}\right) B_{Y}+K,
$$

which means that

$$
\bigcap_{\beta \in \delta_{1} B_{Y}}(\beta+K)+\delta_{2} B_{Y}+K \subseteq\left(\delta_{2}-\delta_{1}\right) B_{Y}+K
$$

(iv). Let $z \in \bigcap_{\beta \in \delta_{1} B_{Y}}(\beta+K), u \in \delta_{2} B_{Y}$ and $\bar{k} \in K$. For any $\varphi \in\left(\delta_{1}-\delta_{2}\right) B_{Y}$, it is clear that $-u+\varphi \in \delta_{1} B_{Y}$. It follows from (3.8) that $z \in-u+\varphi+K$. Thus,

$$
z+u+\bar{k} \in-u+\varphi+K+u+\bar{k} \subseteq \varphi+K .
$$

Due to the arbitrariness of $\varphi \in\left(\delta_{1}-\delta_{2}\right) B_{Y}$, we have $z+u+\bar{k} \in \bigcap_{\beta \in\left(\delta_{1}-\delta_{2}\right) B_{Y}}(\beta+K)$.

Theorem 3.2. Assume that $A_{1}, A_{2}, B_{1}$ and $B_{2}$ are $K$-bounded. Then

$$
h_{K}\left(A_{1}+A_{2}, B_{1}+B_{2}\right) \leq h_{K}\left(A_{1}, B_{1}\right)+h_{K}\left(A_{2}, B_{2}\right) .
$$

Proof. It follows from Lemma 3.1 that $h_{K}\left(A_{1}, B_{1}\right), h_{K}\left(A_{2}, B_{2}\right)$ and $h_{K}\left(A_{1}+A_{2}, B_{1}+B_{2}\right)$ are finite. There are three cases to be considered.

Case 1. $h_{K}\left(A_{1}, B_{1}\right) \geq 0$ and $h_{K}\left(A_{2}, B_{2}\right) \geq 0$. For any $\varepsilon>0$, we conclude from Theorem 3.1 (i) that there exists $h_{K}\left(A_{i}, B_{i}\right) \leq t_{i}<h_{K}\left(A_{i}, B_{i}\right)+\varepsilon$ such that $B_{i} \subseteq A_{i}+K+t_{i} B_{Y}, i=1,2$. Then

$$
B_{1}+B_{2} \subseteq A_{1}+A_{2}+K+K+t_{1} B_{Y}+t_{2} B_{Y} \subseteq A_{1}+A_{2}+K+\left(t_{1}+t_{2}\right) B_{Y} .
$$

Thanks to Proposition 3.1 (i), we have

$$
h_{K}\left(A_{1}+A_{2}, B_{1}+B_{2}\right) \leq t_{1}+t_{2}<h_{K}\left(A_{1}, B_{1}\right)+h_{K}\left(A_{2}, B_{2}\right)+2 \varepsilon .
$$

By the arbitrariness of $\varepsilon>0$, we know that $h_{K}\left(A_{1}+A_{2}, B_{1}+B_{2}\right) \leq h_{K}\left(A_{1}, B_{1}\right)+h_{K}\left(A_{2}, B_{2}\right)$.

Case 2. $h_{K}\left(A_{1}, B_{1}\right)<0$ and $h_{K}\left(A_{2}, B_{2}\right)<0$. For any $\varepsilon>0$ with $h_{K}\left(A_{1}, B_{1}\right)+\varepsilon<0$ and $h_{K}\left(A_{2}, B_{2}\right)+\varepsilon<0$, it follows from Theorem 3.1 (ii) that there exists $h_{K}\left(A_{i}, B_{i}\right) \leq t_{i}<$ 
$h_{K}\left(A_{i}, B_{i}\right)+\varepsilon$ such that $B_{i} \subseteq A_{i}+\bigcap_{\beta \in\left(-t_{i}\right) B_{Y}}(\beta+K), i=1,2$. This together with Lemma 3.3 (ii) implies that

$$
\begin{aligned}
B_{1}+B_{2} & \subseteq A_{1}+A_{2}+\bigcap_{\beta \in\left(-t_{1}\right) B_{Y}}(\beta+K)+\bigcap_{\beta \in\left(-t_{2}\right) B_{Y}}(\beta+K) \\
& \subseteq A_{1}+A_{2}+\bigcap_{\beta \in\left(-t_{1}-t_{2}\right) B_{Y}}(\beta+K) \\
& \subseteq A_{1}+A_{2}+\bigcap_{\beta \in\left(-t_{1}-t_{2}\right) B_{Y}^{0}}(\beta+K) .
\end{aligned}
$$

Due to Proposition 3.3 (i), one has

$$
h_{K}\left(A_{1}+A_{2}, B_{1}+B_{2}\right) \leq t_{1}+t_{2}<h_{K}\left(A_{1}, B_{1}\right)+h_{K}\left(A_{2}, B_{2}\right)+2 \varepsilon .
$$

By the arbitrariness of $\varepsilon>0$, we have $h_{K}\left(A_{1}+A_{2}, B_{1}+B_{2}\right) \leq h_{K}\left(A_{1}, B_{1}\right)+h_{K}\left(A_{2}, B_{2}\right)$.

Case 3. $h_{K}\left(A_{1}, B_{1}\right)<0$ and $h_{K}\left(A_{2}, B_{2}\right) \geq 0$. For any $\varepsilon>0$ with $h_{K}\left(A_{1}, B_{1}\right)+\varepsilon<0$, due to Theorem 3.1 (ii), there exists $h_{K}\left(A_{1}, B_{1}\right) \leq t_{1}<h_{K}\left(A_{1}, B_{1}\right)+\varepsilon$ such that

$$
B_{1} \subseteq A_{1}+\bigcap_{\beta \in\left(-t_{1}\right) B_{Y}}(\beta+K) .
$$

It follows from Theorem 3.1 (i) that there exists $h_{K}\left(A_{2}, B_{2}\right) \leq t_{2}<h_{K}\left(A_{2}, B_{2}\right)+\varepsilon$ such that $B_{2} \subseteq A_{2}+K+t_{2} B_{Y}$. Then

$$
B_{1}+B_{2} \subseteq A_{1}+A_{2}+\bigcap_{\beta \in\left(-t_{1}\right) B_{Y}}(\beta+K)+t_{2} B_{Y}+K .
$$

If $h_{K}\left(A_{1}, B_{1}\right)+h_{K}\left(A_{2}, B_{2}\right) \geq 0$, then $t_{1}+t_{2} \geq h_{K}\left(A_{1}, B_{1}\right)+h_{K}\left(A_{2}, B_{2}\right) \geq 0$, and so $t_{2} \geq-t_{1}>0$. Applying Lemma 3.3 (iii), one has

$$
\bigcap_{\beta \in\left(-t_{1}\right) B_{Y}}(\beta+K)+t_{2} B_{Y}+K \subseteq\left(t_{1}+t_{2}\right) B_{Y}+K .
$$

This together with (3.9) implies that $B_{1}+B_{2} \subseteq A_{1}+A_{2}+\left(t_{1}+t_{2}\right) B_{Y}+K$. By Proposition 3.1 (i), we know that $h_{K}\left(A_{1}+A_{2}, B_{1}+B_{2}\right) \leq t_{1}+t_{2}$.

If $h_{K}\left(A_{1}, B_{1}\right)+h_{K}\left(A_{2}, B_{2}\right)<0$, without loss of generality, we assume that $t_{1}+t_{2}<0$, and so $-t_{1}>t_{2} \geq 0$. Thanks to Lemma 3.3 (iv), we have

$$
\bigcap_{\beta \in\left(-t_{1}\right) B_{Y}}(\beta+K)+t_{2} B_{Y}+K \subseteq \bigcap_{\beta \in\left(-t_{1}-t_{2}\right) B_{Y}}(\beta+K) .
$$

It follows from (3.9) that

$$
B_{1}+B_{2} \subseteq A_{1}+A_{2}+\bigcap_{\beta \in\left(-t_{1}-t_{2}\right) B_{Y}}(\beta+K) \subseteq A_{1}+A_{2}+\bigcap_{\beta \in\left(-t_{1}-t_{2}\right) B_{Y}^{0}}(\beta+K) .
$$

In view of Proposition 3.3 (i), we have $h_{K}\left(A_{1}+A_{2}, B_{1}+B_{2}\right) \leq t_{1}+t_{2}$. Therefore,

$$
h_{K}\left(A_{1}+A_{2}, B_{1}+B_{2}\right) \leq t_{1}+t_{2}<h_{K}\left(A_{1}, B_{1}\right)+h_{K}\left(A_{2}, B_{2}\right)+2 \varepsilon \text {. }
$$

By the arbitrariness of $\varepsilon>0$, we have

$$
h_{K}\left(A_{1}+A_{2}, B_{1}+B_{2}\right) \leq h_{K}\left(A_{1}, B_{1}\right)+h_{K}\left(A_{2}, B_{2}\right) .
$$

This completes the proof. 
Theorem 3.3. Assume that $A, B$ and $D$ are $K$-bounded. Then

(i) $h_{K}(\operatorname{co} A, \operatorname{co} B) \leq h_{K}(A, B)$;

(ii) $h_{K}(A+D, B+D) \leq h_{K}(A, B)$.

Proof. (i). Let $\eta=h_{K}(A, B)$. There are two cases to be considered.

Case 1. $\eta \geq 0$. For any $\varepsilon>0$, it follows from Theorem 3.1 (i) that there exists $\eta \leq \bar{t}<\eta+\varepsilon$ such that $B \subseteq A+K+\bar{t} B_{Y} \subseteq \operatorname{co} A+K+\bar{t} B_{Y}$. Noting that $\operatorname{co} A+K+\bar{t} B_{Y}$ is convex, we have $\operatorname{co} B \subseteq \operatorname{co} A+K+\bar{t} B_{Y}$. By Proposition 3.1 (i), we have $h_{K}(\operatorname{co} A, \operatorname{co} B) \leq \bar{t}<\eta+\varepsilon=h_{K}(A, B)+\varepsilon$.

Case 2. $\eta<0$. For any $\varepsilon>0$, we conclude from Theorem 3.1 (ii) that there exists $t_{0}<0$ such that $\eta \leq t_{0}<\eta+\varepsilon$ and

$$
B \subseteq A+\bigcap_{\beta \in\left(-t_{0}\right) B_{Y}}(\beta+K) \subseteq \operatorname{co} A+\bigcap_{\beta \in\left(-t_{0}\right) B_{Y}}(\beta+K) .
$$

Since $\operatorname{co} A+\bigcap_{\beta \in\left(-t_{0}\right) B_{Y}}(\beta+K)$ is convex, we have $\operatorname{co} B \subseteq \operatorname{co} A+\bigcap_{\beta \in\left(-t_{0}\right) B_{Y}}(\beta+K)$. This together with Proposition 3.3 (i) implies that

$$
h_{K}(\operatorname{co} A, \operatorname{co} B) \leq t_{0}<\eta+\varepsilon=h_{K}(A, B)+\varepsilon .
$$

Hence, it follows from the arbitrariness of $\varepsilon>0$ that $h_{K}(\operatorname{co} A, \operatorname{co} B) \leq h_{K}(A, B)$.

(ii). Similar to the proof of (i), we can prove that (ii) holds.

\section{Dini Directional Derivatives with Applications in Set Optimization PROBLEMS}

In this section, we investigate the Dini directional derivatives for set-valued mappings and apply them to derive some necessary and sufficient optimality conditions for set optimization problems.

Based on the results obtained in Section 3, we have the basic ingredients to define the upper and lower Dini directional derivatives for set-valued mappings. Thus, we can give the following definition.

Definition 4.1. Let $F: X \rightrightarrows Y$ be a set-valued mapping and $x, u \in X$. The upper and lower Dini directional derivative of $F$ at $x$ in direction $u$ are, respectively, defined by

$$
F^{\uparrow}(x, u)=\limsup _{t \downarrow 0} \frac{1}{t} h_{K}(F(x+t u), F(x))=\inf _{0<s} \sup _{0<t \leq s} \frac{1}{t} h_{K}(F(x+t u), F(x)),
$$

and

$$
F^{\downarrow}(x, u)=\liminf _{t \downarrow 0} \frac{1}{t} h_{K}(F(x+t u), F(x))=\sup _{0<s} \inf _{0<t \leq s} \frac{1}{t} h_{K}(F(x+t u), F(x)) .
$$

If both derivatives coincide, then $F^{\prime}(x, u)=F^{\uparrow}(x, u)=F^{\downarrow}(x, u)$ is the Dini directional derivative of $F$ at $x$ in direction $u$.

Clearly, $F^{\uparrow}(x, u) \geq F^{\downarrow}(x, u)$. Thus, we know that $F^{\prime}(x, u)$ exists if and only if $F^{\uparrow}(x, u) \leq$ $F^{\downarrow}(x, u)$.

Theorem 4.1. Assume that $F$ is $K$-convex on $X$ with nonempty and $K$-bounded values. Then

(i) the Dini derivative of $F$ at $x \in X$ exists for all $u \in X$ and

$$
F^{\prime}(x, u)=F^{\uparrow}(x, u)=F^{\downarrow}(x, u)=\inf _{0<s} \frac{1}{s} h_{K}(F(x+s u), F(x)) ;
$$


(ii) for any given $x, u \in X, F^{\prime}(x, \xi u)=\xi F^{\prime}(x, u)$ for all $\xi>0$;

(iii) for any given $x \in X, F^{\prime}(x, \cdot)$ is a convex function, i.e., for any $u_{1}, u_{2} \in X$ and $\lambda \in[0,1]$,

$$
F^{\prime}\left(x, \lambda u_{1}+(1-\lambda) u_{2}\right) \leq \lambda F^{\prime}\left(x, u_{1}\right)+(1-\lambda) F^{\prime}\left(x, u_{2}\right) .
$$

Proof. (i). We first prove that, for any $t, r \in \mathbb{R}$ with $0<t \leq r$,

$$
\frac{1}{t} h_{K}(F(x+t u), F(x)) \leq \frac{1}{r} h_{K}(F(x+r u), F(x)) .
$$

As $F$ is $K$-convex on $X$, one has

$$
\frac{r-t}{r} F(x)+\frac{t}{r} F(x+r u) \subseteq F(x+t u)+K .
$$

Let $\eta:=h_{K}(F(x+r u), F(x))$. There are two cases to be considered.

Case 1. $\eta \geq 0$. For any $\varepsilon>0$, there exists $\delta \in \mathbb{R}$ such that $\eta<\delta \leq \eta+\varepsilon$. Thanks to Proposition 3.2 (i) and $h_{K}(F(x+r u), F(x))=\eta<\delta$, we have

$$
F(x) \subseteq F(x+r u)+K+\delta B_{Y}^{0} \subseteq F(x+r u)+K+\delta B_{Y} .
$$

For any $y \in F(x)$, it follows from (4.3) that there exist $z_{r} \in F(x+r u), k_{0} \in K$ and $b_{0} \in B_{Y}$ such that $y=z_{r}+k_{0}+\delta b_{0}$. In view of (4.2), there exist $z_{t} \in F(x+t u)$ and $\bar{k} \in K$ satisfying

$$
\frac{r-t}{r} y+\frac{t}{r} z_{r}=z_{t}+\bar{k} .
$$

Applying (4.4) and $y=z_{r}+k_{0}+\delta b_{0}$, one has

$$
\begin{aligned}
y & =\frac{r-t}{r} y+\frac{t}{r} y=\frac{r-t}{r} y+\frac{t}{r} z_{r}+\frac{t}{r} k_{0}+\frac{t}{r} \delta b_{0} \\
& =z_{t}+\bar{k}+{ }^{t} k_{0}+{ }_{-}^{t} \delta b_{0} \in F(x+t u)+K+{ }_{-}^{t} \delta B_{Y} .
\end{aligned}
$$

By the arbitrariness of $y \in F(x)$, we know that $F(x) \subseteq F(x+t u)+K+\frac{t}{r} \delta B_{Y}$. This together with Proposition 3.1 (i) implies that

$$
h_{K}(F(x+t u), F(x)) \leq \frac{t}{r} \delta \leq \frac{t}{r}(\eta+\varepsilon) .
$$

Due to the arbitrariness of $\varepsilon>0$, we have $h_{K}(F(x+t u), F(x)) \leq \frac{t}{r} \eta$ and so (4.1) holds.

Case 2. $\eta<0$. For any $\varepsilon>0$ with $\eta+\varepsilon<0$, there exists $\delta>0$ such that $\eta<-\delta \leq \eta+\varepsilon$. It follows from Proposition 3.4 (i) that

$$
F(x) \subseteq F(x+r u)+\bigcap_{\beta \in \delta B_{Y}}(\beta+\operatorname{int} K) .
$$

For any $y \in F(x)$, due to (4.5), there exist $z_{r} \in F(x+r u)$ and $v_{0} \in \bigcap_{\beta \in \delta B_{Y}}(\beta+\operatorname{int} K)$ satisfying $y=z_{r}+v_{0}$. By (4.2), there exist $z_{t} \in F(x+t u)$ and $k_{0} \in K$ such that

$$
\frac{r-t}{r} y+\frac{t}{r} z_{r}=z_{t}+k_{0} .
$$

It follows from (4.6), $y=z_{r}+v_{0}$ and Lemma 3.3 (i) that

$$
\begin{aligned}
y & =\frac{r-t}{r} y+\frac{t}{r} z_{r}+\frac{t}{r} v_{0}=z_{t}+k_{0}+\frac{t}{r} v_{0} \in F(x+t u)+K+\frac{t}{r} \bigcap_{\beta \in \delta B_{Y}}(\beta+\operatorname{int} K) \\
& \subseteq F(x+t u)+\bigcap_{\beta \in \frac{t}{r} \delta B_{Y}}(\beta+\operatorname{int} K) .
\end{aligned}
$$


By the arbitrariness of $y \in F(x)$, we have

$$
F(x) \subseteq F(x+t u)+\bigcap_{\beta \in \frac{t}{r} \delta B_{Y}}(\beta+\operatorname{int} K) .
$$

This together with Proposition 3.3 (i) implies that

$$
h_{K}(F(x+t u), F(x)) \leq \frac{t}{r}(-\delta) \leq \frac{t}{r}(\eta+\varepsilon) .
$$

By the arbitrariness of $\varepsilon>0$, we have $h_{K}(F(x+t u), F(x)) \leq \frac{t}{r} \eta$, and so (4.1) holds.

We conclude from (4.1) that, for any $s>0$,

$$
\sup _{0<t \leq s} \frac{1}{t} h_{K}(F(x+t u), F(x))=\frac{1}{s} h_{K}(F(x+s u), F(x))
$$

and so

$$
F^{\uparrow}(x, u)=\inf _{0<s} \frac{1}{S} h_{K}(F(x+s u), F(x)) .
$$

We claim that, for any $s>0$,

$$
\inf _{0<t \leq s} \frac{1}{t} h_{K}(F(x+t u), F(x))=\inf _{0<r} \frac{1}{r} h_{K}(F(x+r u), F(x)) .
$$

It is clear that

$$
\inf _{0<t \leq s} \frac{1}{t} h_{K}(F(x+t u), F(x)) \geq \inf _{0<r} \frac{1}{r} h_{K}(F(x+r u), F(x)) .
$$

Suppose that there exists $\zeta \in \mathbb{R}$ such that

$$
\inf _{0<t \leq s} \frac{1}{t} h_{K}(F(x+t u), F(x))>\zeta>\inf _{0<r} \frac{1}{r} h_{K}(F(x+r u), F(x)) .
$$

Then there exists $r_{0}>0$ such that

$$
\frac{1}{r_{0}} h_{K}\left(F\left(x+r_{0} u\right), F(x)\right)<\zeta .
$$

If $0<r_{0} \leq s$, then

$$
\frac{1}{r_{0}} h_{K}\left(F\left(x+r_{0} u\right), F(x)\right) \geq \inf _{0<t \leq s} \frac{1}{t} h_{K}(F(x+t u), F(x))>\zeta,
$$

which contradicts (4.8). Thus, $r_{0}>s$. It follows from (4.1) that

$$
\frac{1}{r_{0}} h_{K}\left(F\left(x+r_{0} u\right), F(x)\right) \geq \frac{1}{s} h_{K}(F(x+s u), F(x)) \geq \inf _{0<t \leq s} \frac{1}{t} h_{K}(F(x+t u), F(x))>\zeta,
$$

which contradicts (4.8). Therefore, we know that (4.7) holds and so

$$
F^{\downarrow}(x, u)=\sup _{0<s} \inf _{0<t \leq s} \frac{1}{t} h_{K}(F(x+t u), F(x))=\inf _{0<r} \frac{1}{r} h_{K}(F(x+r u), F(x)) .
$$

(ii). For any $\xi>0$, one has

$$
F^{\prime}(x, \xi u)=\inf _{0<s} \frac{1}{s} h_{K}(F(x+s \xi u), F(x)) .
$$

Let $r=s \xi$. Then $\frac{1}{s}=\frac{\xi}{r}$ and $s>0 \Leftrightarrow r>0$. In view of (4.9), we have

$$
F^{\prime}(x, \xi u)=\inf _{0<r} \frac{\xi}{r} h_{K}(F(x+r u), F(x))=\xi \inf _{0<r} \frac{1}{r} h_{K}(F(x+r u), F(x))=\xi F^{\prime}(x, u) .
$$


(iii). Let $u_{1}, u_{2} \in X$ and $\lambda \in(0,1)$. For any $\varepsilon>0$, noting that

$$
F^{\prime}\left(x, u_{i}\right)=\inf _{0<s} \frac{1}{s} h_{K}\left(F\left(x+s u_{i}\right), F(x)\right), i=1,2,
$$

there exist $s_{1}>0$ and $s_{2}>0$ such that

$$
\frac{1}{s_{i}} h_{K}\left(F\left(x+s_{i} u_{i}\right), F(x)\right)<F^{\prime}\left(x, u_{i}\right)+\varepsilon, i=1,2 .
$$

Let $s_{0}=\min \left\{s_{1}, s_{2}\right\}>0$. In view of (4.1) and (4.10), we can find $\delta_{1}, \delta_{2} \in \mathbb{R}$ satisfying

$$
\frac{1}{s_{0}} h_{K}\left(F\left(x+s_{0} u_{1}\right), F(x)\right) \leq \frac{1}{s_{1}} h_{K}\left(F\left(x+s_{1} u_{1}\right), F(x)\right)<\delta_{1}<F^{\prime}\left(x, u_{1}\right)+\varepsilon
$$

and

$$
\frac{1}{s_{0}} h_{K}\left(F\left(x+s_{0} u_{2}\right), F(x)\right) \leq \frac{1}{s_{2}} h_{K}\left(F\left(x+s_{2} u_{2}\right), F(x)\right)<\delta_{2}<F^{\prime}\left(x, u_{2}\right)+\varepsilon .
$$

Noting that $F$ is $K$-convex on $X$ and

$$
\lambda\left(x+s_{0} u_{1}\right)+(1-\lambda)\left(x+s_{0} u_{2}\right)=x+s_{0}\left(\lambda u_{1}+(1-\lambda) u_{2}\right),
$$

we have

$$
\lambda F\left(x+s_{0} u_{1}\right)+(1-\lambda) F\left(x+s_{0} u_{2}\right) \subseteq F\left(x+s_{0}\left(\lambda u_{1}+(1-\lambda) u_{2}\right)\right)+K .
$$

There are three cases to be considered.

Case 1. $F^{\prime}\left(x, u_{1}\right) \geq 0$ and $F^{\prime}\left(x, u_{2}\right) \geq 0$. It follows from (4.11), (4.12) and Proposition 3.2 (i) that

$$
F(x) \subseteq F\left(x+s_{0} u_{i}\right)+K+s_{0} \delta_{i} B_{Y}^{0}, \quad i=1,2 .
$$

This together with (4.13) implies that

$$
\begin{aligned}
F(x) \subseteq & \lambda F(x)+(1-\lambda) F(x) \subseteq \lambda F\left(x+s_{0} u_{1}\right)+\lambda K+\lambda s_{0} \delta_{1} B_{Y}^{0} \\
& +(1-\lambda) F\left(x+s_{0} u_{2}\right)+(1-\lambda) K+(1-\lambda) s_{0} \delta_{2} B_{Y}^{0} \\
\subseteq & F\left(x+s_{0}\left(\lambda u_{1}+(1-\lambda) u_{2}\right)\right)+K+s_{0}\left(\lambda \delta_{1}+(1-\lambda) \delta_{2}\right) B_{Y}^{0} .
\end{aligned}
$$

We conclude from Proposition 3.1 (i) that

$$
h_{K}\left(F\left(x+s_{0}\left(\lambda u_{1}+(1-\lambda) u_{2}\right)\right), F(x)\right) \leq s_{0}\left(\lambda \delta_{1}+(1-\lambda) \delta_{2}\right) .
$$

Applying (4.11), (4.12) and (4.14), one has

$$
\begin{aligned}
F^{\prime}\left(x, \lambda u_{1}+(1-\lambda) u_{2}\right) & \leq \frac{1}{s_{0}} h_{K}\left(F\left(x+s_{0}\left(\lambda u_{1}+(1-\lambda) u_{2}\right)\right), F(x)\right) \\
& \leq \lambda \delta_{1}+(1-\lambda) \delta_{2} \\
& <\lambda F^{\prime}\left(x, u_{1}\right)+(1-\lambda) F^{\prime}\left(x, u_{2}\right)+\varepsilon .
\end{aligned}
$$

By the arbitrariness of $\varepsilon>0$, it follows from (4.15) that

$$
F^{\prime}\left(x, \lambda u_{1}+(1-\lambda) u_{2}\right) \leq \lambda F^{\prime}\left(x, u_{1}\right)+(1-\lambda) F^{\prime}\left(x, u_{2}\right) .
$$

Case 2. $F^{\prime}\left(x, u_{1}\right)<0$ and $F^{\prime}\left(x, u_{2}\right)<0$. Without loss of generality, we can assume that $\delta_{1}<F^{\prime}\left(x, u_{1}\right)+\varepsilon<0$ and $\delta_{2}<F^{\prime}\left(x, u_{2}\right)+\varepsilon<0$. By (4.11), (4.12) and Proposition 3.4 (i), we obtain

$$
F(x) \subseteq F\left(x+s_{0} u_{i}\right)+\bigcap_{\beta \in s_{0}\left(-\delta_{i}\right) B_{Y}}(\beta+\operatorname{int} K), i=1,2 .
$$


This together with (4.13) and Lemma 3.3 (i) implies that

$$
\begin{aligned}
F(x) \subseteq & \lambda F(x)+(1-\lambda) F(x) \subseteq \lambda F\left(x+s_{0} u_{1}\right)+\lambda \bigcap_{\beta \in s_{0}\left(-\delta_{1}\right) B_{Y}}(\beta+\operatorname{int} K) \\
& +(1-\lambda) F\left(x+s_{0} u_{2}\right)+(1-\lambda) \bigcap_{\beta \in s_{0}\left(-\delta_{2}\right) B_{Y}}(\beta+\operatorname{int} K) \\
\subseteq & F\left(x+s_{0}\left(\lambda u_{1}+(1-\lambda) u_{2}\right)\right)+K+\bigcap_{\beta \in s_{0}\left(\lambda\left(-\delta_{1}\right)+(1-\lambda)\left(-\delta_{2}\right)\right) B_{Y}}(\beta+\operatorname{int} K) \\
\subseteq & F\left(x+s_{0}\left(\lambda u_{1}+(1-\lambda) u_{2}\right)\right)+\bigcap_{\beta \in s_{0}\left(\lambda\left(-\delta_{1}\right)+(1-\lambda)\left(-\delta_{2}\right)\right) B_{Y}}(\beta+\operatorname{int} K)
\end{aligned}
$$

In view of Proposition 3.3 (i), one has

$$
h_{K}\left(F\left(x+s_{0}\left(\lambda u_{1}+(1-\lambda) u_{2}\right)\right), F(x)\right) \leq s_{0}\left(\lambda \delta_{1}+(1-\lambda) \delta_{2}\right) .
$$

Thus, it is easy to see that (4.16) holds.

Case 3. $F^{\prime}\left(x, u_{1}\right) \geq 0$ and $F^{\prime}\left(x, u_{2}\right)<0$. Without loss of generality, we can assume that $\delta_{2}<F^{\prime}\left(x, u_{2}\right)+\varepsilon<0$. Due to (4.11) and Proposition 3.2 (i), one has

$$
F(x) \subseteq F\left(x+s_{0} u_{1}\right)+K+s_{0} \delta_{1} B_{Y}^{0} \subseteq F\left(x+s_{0} u_{1}\right)+K+s_{0} \delta_{1} B_{Y} .
$$

We conclude from (4.12) and Proposition 3.4 (i) that

$$
F(x) \subseteq F\left(x+s_{0} u_{2}\right)+\bigcap_{\beta \in s_{0}\left(-\delta_{2}\right) B_{Y}}(\beta+\mathrm{int} K) \subseteq F\left(x+s_{0} u_{2}\right)+\bigcap_{\beta \in s_{0}\left(-\delta_{2}\right) B_{Y}}(\beta+K) .
$$

Applying (4.13), (4.17), (4.18) and Lemma 3.3 (i), we obtain

$$
\begin{aligned}
F(x) \subseteq & \lambda F(x)+(1-\lambda) F(x) \subseteq \lambda F\left(x+s_{0} u_{1}\right)+\lambda K+\lambda s_{0} \delta_{1} B_{Y} \\
& +(1-\lambda) F\left(x+s_{0} u_{2}\right)+(1-\lambda) \bigcap_{\beta \in s_{0}\left(-\delta_{2}\right) B_{Y}}(\beta+K) \\
\subseteq & F\left(x+s_{0}\left(\lambda u_{1}+(1-\lambda) u_{2}\right)\right)+K+\lambda s_{0} \delta_{1} B_{Y} \\
& +\bigcap_{\beta \in s_{0}(1-\lambda)\left(-\delta_{2}\right) B_{Y}}(\beta+K) .
\end{aligned}
$$

If $\lambda s_{0} \delta_{1} \geq(1-\lambda) s_{0}\left(-\delta_{2}\right)>0$, then it follows from (4.19) and Lemma 3.3 (iii) that

$$
F(x) \subseteq F\left(x+s_{0}\left(\lambda u_{1}+(1-\lambda) u_{2}\right)\right)+K+\left(\lambda s_{0} \delta_{1}+(1-\lambda) s_{0} \delta_{2}\right) B_{Y} .
$$

This together with Proposition 3.1 (i) implies that

$$
h_{K}\left(F\left(x+s_{0}\left(\lambda u_{1}+(1-\lambda) u_{2}\right)\right), F(x)\right) \leq s_{0}\left(\lambda \delta_{1}+(1-\lambda) \delta_{2}\right) .
$$

If $0<\lambda s_{0} \delta_{1}<(1-\lambda) s_{0}\left(-\delta_{2}\right)$, we conclude from (4.19) and Lemma 3.3 (iv) that

$$
F(x) \subseteq F\left(x+s_{0}\left(\lambda u_{1}+(1-\lambda) u_{2}\right)\right)+K+\bigcap_{\beta \in\left(\lambda s_{0}\left(-\delta_{1}\right)+(1-\lambda) s_{0}\left(-\delta_{2}\right)\right) B_{Y}}(\beta+K) .
$$

In view of Proposition 3.3 (i), we have

$$
h_{K}\left(F\left(x+s_{0}\left(\lambda u_{1}+(1-\lambda) u_{2}\right)\right), F(x)\right) \leq s_{0}\left(\lambda \delta_{1}+(1-\lambda) \delta_{2}\right) .
$$

Therefore, we can see that (4.15) holds. By the arbitrariness of $\varepsilon>0$, it follows from (4.15) that

$$
F^{\prime}\left(x, \lambda u_{1}+(1-\lambda) u_{2}\right) \leq \lambda F^{\prime}\left(x, u_{1}\right)+(1-\lambda) F^{\prime}\left(x, u_{2}\right) .
$$


This completes the proof.

Remark 4.1. It is worth mentioning that Theorem 4.1 is different from Theorems 4.1 and 4.2 of [35]. Moreover, when we study the nonlinear scalarizing function $h_{K}(\cdot, \cdot)$, it is hard to avoid considering the case that $h_{K}(\cdot, \cdot)$ is negative, which makes the research work more difficult. In fact, dealing with $h_{K}(\cdot, \cdot)$ is more difficult than dealing with the nonlinear scalarizing function $G_{e}(\cdot, \cdot)$ introduced by Hernández and Rodríguez-Marín [14].

Theorem 4.2. Assume that $D$ is convex and $F$ is $K$-convex on $D$ with nonempty and $K$-bounded values. For any given $x_{0} \in D$, if $F^{\prime}\left(x_{0}, u\right)>0$ for all $u \in X$ with $x_{0}+u \in D$ and $u \neq 0$, then $x_{0} \in E_{l}(F, D)$.

Proof. For any $y \in D$ with $y \neq x_{0}$, by the assumption, we have $F^{\prime}\left(x_{0}, y-x_{0}\right)>0$. It follows from Theorem 4.1 (i) that

$$
0<F^{\prime}\left(x_{0}, y-x_{0}\right)=\inf _{0<s} \frac{1}{s} h_{K}\left(F\left(x_{0}+s\left(y-x_{0}\right)\right), F\left(x_{0}\right)\right) \leq h_{K}\left(F(y), F\left(x_{0}\right)\right) .
$$

In view of Corollary 3.1 (i), we obtain that $F(y) \not^{l} F\left(x_{0}\right)$ for all $y \in D$ with $y \neq x_{0}$. This means that $x_{0} \in E_{l}(F, D)$.

Theorem 4.3. Assume that $D$ is convex and $F$ is $K$-convex on $D$ with nonempty and $K$-bounded values. Let $x_{0} \in D$ such that $F\left(x_{0}\right)$ is $K$-compact. Then, $x_{0} \in W_{l}(F, D)$ if and only if $F^{\prime}\left(x_{0}, u\right) \geq 0$ for all $u \in X$ with $x_{0}+u \in D$.

Proof. We first prove the sufficiency. For any $y \in D$, it follows that $F^{\prime}\left(x_{0}, y-x_{0}\right) \geq 0$. Due to Theorem 4.1 (i), we have

$$
0 \leq F^{\prime}\left(x_{0}, y-x_{0}\right)=\inf _{0<s} \frac{1}{s} h_{K}\left(F\left(x_{0}+s\left(y-x_{0}\right)\right), F\left(x_{0}\right)\right) \leq h_{K}\left(F(y), F\left(x_{0}\right)\right) .
$$

By employing Theorem 4.11 of [33], we know that, for any $y \in D, F(y) \ll^{l} F\left(x_{0}\right)$ is not true and so $x_{0} \in W_{l}(F, D)$.

Next, we show the necessity. Suppose that $x_{0} \in W_{l}(F, D)$. Then it follows from Lemma 2.5 that there does not exist $y \in D$ satisfying $F(y) \ll^{l} F\left(x_{0}\right)$. This together with Theorem 4.11 of [33] implies that

$$
h_{K}\left(F(y), F\left(x_{0}\right)\right) \geq 0, \quad \forall y \in D .
$$

For any $u \in X$ with $x_{0}+u \in D$ and for any $\lambda \in(0,1]$, the convexity of $D$ implies that $x_{0}+\lambda u \in D$. By (4.20), we have $\frac{1}{\lambda} h_{K}\left(F\left(x_{0}+\lambda u\right), F\left(x_{0}\right)\right) \geq 0$ and so

$$
\inf _{0<\lambda \leq 1} \frac{1}{\lambda} h_{K}\left(F\left(x_{0}+\lambda u\right), F\left(x_{0}\right)\right) \geq 0 .
$$

Applying (4.7), (4.21) and Theorem 4.1 (i), we have

$$
F^{\prime}\left(x_{0}, u\right)=\inf _{0<r} \frac{1}{r} h_{K}\left(F\left(x_{0}+r u\right), F\left(x_{0}\right)\right)=\inf _{0<\lambda \leq 1} \frac{1}{\lambda} h_{K}\left(F\left(x_{0}+\lambda u\right), F\left(x_{0}\right)\right) \geq 0 .
$$

This completes the proof.

Remark 4.2. The proofs of Theorems 4.2 and 4.3 are similar to the proof of Theorem 4.3 in [35]. However, it is worth pointing out that Theorems 4.2 and 4.3 are under weaker conditions. In fact, Theorems 4.2 and 4.3 do not need to assume that $F(\cdot)$ is locally $K$-Lipschitz $C$-continuous with respect to $e \in-\operatorname{int} C$ at $x_{0} \in D$. 


\section{CONCLUSIONS}

The paper dealt with the set scalarization function and the Dini directional derivatives for set-valued mappings with applications to set optimization problems. The main contributions are as follows: (i) we employed the properties of the (oriented) distance function to show some properties of the set scalarization function with negative values; (ii) we investigated the Dini directional derivatives for set-valued mappings by using the set scalarization function; (iii) we applied the Dini directional derivatives to derive some necessary and sufficient optimality conditions for set optimization problems.

It is well known that the study of the connectedness, the well-posedness, and the stability of the solution sets is of great interest in set optimization problems. However, to the best of our knowledge, the connectedness, the well-posedness, and the stability of the solution sets for set optimization problems via the set scalarization function introduced by $\mathrm{Ha}$ [31] have not been explored until now. Thus, several interesting issues may deserve further research. It may be interesting to explore the connectedness, the well-posedness, and the stability of the solution sets for set optimization problems by using the set scalarization function. We leave these as our future work.

\section{Acknowledgements}

The authors are grateful to Professor Jen-Chih Yao for his valuable comments and suggestions. This work was supported by the National Natural Science Foundation of China (11801257, 11471230 , and 11671282). This work was also supported by the Ministry of Science and Technology, Taiwan under Grant Number 110-2115-M-037-001.

\section{REFERENCES}

[1] A.A. Khan, C. Tammer, C. Zălinescu, Set-Valued Optimization. An Introduction with Applications, Springer, Berlin, 2015.

[2] D.S. Kim, B.S. Mordukhovich, T.S. Pham, N.V. Tuyen, Existence of efficient and properly efficient solutions to problems of constrained vector optimization, Math. Program. DOI: 10.1007/s10107-020-01532-y.

[3] A.H. Hamel, F. Heyde, Duality for set-valued measures of risk, SIAM J. Finan. Math. 1 (2010), 66-95.

[4] E. Köbis, C. Tammer, J.C. Yao, Optimality conditions for set-valued optimization problems based on set approach and applications in uncertain optimization. J. Nonlinear Convex Anal. 18 (2017), 1001-1014.

[5] C.L. Zhang, N.J. Huang, On the stability of minimal solutions for parametric set optimization problems, Appl. Anal. 100 (2021), 1533-1543.

[6] C.L. Zhang, N.J. Huang, Well-posedness and stability in set optimization with applications, Positivity 25 (2021), 1153-1173.

[7] C.L. Zhang, L.W. Zhou, N.J. Huang, Stability of minimal solution mappings for parametric set optimization problems with pre-order relations, Pacific J. Optim. 15 (2019), 491-504.

[8] C. Gerth (Tammer), P. Weidner, Nonconvex separation theorems and some applications in vector optimization, J. Optim. Theory Appl. 67 (1990), 297-320.

[9] J. Jahn, Vector optimization. Theory, Applications, and Extensions, 2nd ed. Springer, Berlin, 2011.

[10] D.T. Luc, Theory of Vector Optimization, Lecture Notes in Economics and Mathematical Systems, vol. 319, Springer, Berlin 1989.

[11] E. Miglierina, E. Molho, Scalarization and stability in vector optimization, J. Optim. Theory Appl. 114 (2002), 657-670.

[12] Y. Han, N.J. Huang, Continuity and convexity of a nonlinear scalarizing function in set optimization problems with applications, J. Optim. Theory Appl. 177 (2018), 679-695.

[13] Y. Han, Nonlinear scalarizing functions in set optimization problems, Optimization 68 (2019), $1685-1718$. 
[14] E. Hernández, L. Rodríguez-Marín, Nonconvex scalarization in set optimization with set-valued maps, J. Math. Anal. Appl. 325 (2007), 1-18.

[15] Khushboo, C.S. Lalitha, Scalarizations for a set optimization problem using generalized oriented distance function, Positivity 23 (2019), 1195-1213.

[16] S.Y. Cho, A monotone Bregan projection algorithm for fixed point and equilibrium problems in a reflexive Banach space, Filomat, 34 (2020), 1487-1497.

[17] Y.D. Xu, S.J. Li, A new nonlinear scalarization function and applications. Optimization 65 (2016), $207-231$.

[18] C. Gerstewitz(Tammer), Nichtkonvexe Dualität in der Vektoroptimierung, Wissenschaftliche Zeitschrift der TH Leuna-Merseburg, 25 (1983), 357-364.

[19] G.Y. Chen, X.Q. Yang, H. Yu, A nonlinear scalarization function and generalized quasi-vector equilibrium problems, J. Glob. Optim. 32 (2005), 451-466.

[20] C. Gerstewitz (Tammer), E. Iwanow, Dualität für nichtkonvexe Vektoroptimierungsprobleme, Wiss. Z. Tech. Hochsch. Ilmenau, 2 (1985), 61-81.

[21] A. Göpfert, C. Tammer, C. Zălinescu, On the vectorial Ekeland's variational principle and minimal points in product spaces, Nonlinear Anal. TMA 39 (2000), 909-922.

[22] J.B. Hiriart-Urruty, Tangent cone, generalized gradients and mathematical programming in Bananch spaces, Math. Oper. Res. 4 (1979), 79-97.

[23] J.B. Hiriart-Urruty, New concepts in nondifferentiable programming, Bull. Soc. Math. France Mém. 60 (1979), 57-85.

[24] M. Durea, J. Dutta, C. Tammer, Lagrange multipliers for $\varepsilon$-pareto solutions in vector optimization with nonsolid cones in Banach spaces, J. Optim. Theory Appl. 145 (2010), 196-211.

[25] Y. Gao, X.M. Yang, Properties of the nonlinear scalar functional and its applications to vector optimization problems, J. Glob. Optim. 73 (2019), 869-889.

[26] C.G. Liu, K.F. Ng, W.H. Yang, Merit functions in vector optimization, Math. Program. 119 (2009), $215-237$.

[27] E. Miglierina, E. Molho, M. Rocca, Well-posedness and scalarization in vector optimization, J. Optim. Theory Appl. 126 (2005), 391-409.

[28] A. Zaffaroni, Degrees of efficiency and degrees of minimality, SIAM J. Control Optim. 42 (2003), 1071-1086.

[29] J.W. Chen, Q.H. Ansari, J.C. Yao, Characterizations of set order relations and constrained set optimization problems via oriented distance function, Optimization 66 (2017), 1741-1754.

[30] Q.H. Ansari, E. Köbis, P.K. Sharma, Characterizations of set relations with respect to variable domination structures via oriented distance function, Optimization 67 (2018), 1389-1407.

[31] T.X.D. Ha, A Hausdorff-type distance, a directional derivative of a set-valued map and applications in set optimization, Optimization 67 (2018), 1031-1050.

[32] B. Jiménez, V. Novo, A. Vílchez, A set scalarization function based on the oriented distance and relations with other set scalarizations, Optimization 67 (2018), 2091-2116.

[33] B. Jiménez, V. Novo, A. Vílchez, Characterization of set relations through extensions of the oriented distance, Math. Meth. Oper. Res. 91 (2020), 89-115.

[34] B. Jiménez, V. Novo, A. Vílchez, Six set scalarizations based on the oriented distance: properties and application to set optimization, Optimization 69 (2020), 437-470.

[35] Y. Han, A Hausdorff-type distance, the Clarke generalized directional derivative and applications in set optimization problems, Appl. Anal. DOI: 10.1080/00036811.2020.1778673.

[36] G. Giorgi, S. Komlósi, Dini derivatives in optimization-Part I, Decis. Econ. Finance. 15 (1992), 3-30.

[37] A. Göpfert, H. Riahi, C. Tammer, C. Zălinescu, Variational Methods in Partially Ordered Spaces, Springer, Berlin, 2003.

[38] K. Kuratowski, Topology, Vols. 1 and 2. Academic Press, New York, 1968. 